\title{
Immunohistochemical and clinicopathological correlation of the metastasis-associated gene 1 (MTA1) expression in benign and malignant pancreatic endocrine tumors
}

\author{
Matthias D Hofer ${ }^{1,2,3}$, Martin C Chang ${ }^{1,2,3}$, Kelly A Hirko ${ }^{1}$, Mark A Rubin ${ }^{1,2}$ \\ and Vânia Nosé ${ }^{1,2}$ \\ ${ }^{1}$ Department of Pathology, Brigham and Women's Hospital, Boston, MA, USA and ${ }^{2}$ Harvard Medical School, \\ Boston, MA, USA
}

\begin{abstract}
Pancreatic endocrine tumors are rare tumors with unpredictable clinical behavior. No histological features or immunohistochemical markers reliably predict malignant progression and the molecular basis of progression of pancreatic endocrine tumors remains unknown. The metastasis-associated gene 1 is thought to play a role in transcription repression and estrogen receptor interaction and is overexpressed in several human cancers, including endocrine neoplasms. The purpose of this study was to analyze the expression of metastasisassociated gene 1 in pancreatic endocrine tumors for its possible role in malignant progression. Twenty-seven pancreatic endocrine tumors were identified from our archive. The mean age at presentation was $\mathbf{5 7}$ years (range 28-86); the male/female ratio was 1.25 to 1 , and the mean size was $4.5 \mathrm{~cm}(0.1-18 \mathrm{~cm})$. The clinical followup data were examined and tumors were classified according to the $\mathbf{2 0 0 4}$ World Health Organization criteria as benign behavior (WHO 1.1), uncertain behavior (WHO 1.2), well-differentiated endocrine carcinoma (WHO 2), and poorly differentiated endocrine carcinoma (WHO 3). Histopathological and immunohistochemical stains were evaluated and metastasis-associated gene 1 expression scored semiquantitatively as absent $(1+)$, weak $(2+)$, moderate $(3+)$, or strong $(4+)$. Statistical analysis was performed using Kruskal-Wallis nonparametric analysis of variance with a significance level of 0.05 . Metastasis-associated gene 1 expression was significantly higher in malignant tumors $(n=17)$ with a mean staining intensity of 3.8 compared with 2.9 in benign tumors $(n=10, P=0.046)$. The expression levels were significantly associated with WHO class $(P=0.028)$, as well as size of tumor $(P=0.029)$, and mitotic rate $(P=0.035)$. Metastasis-associated gene 1 expression was associated with local invasion with borderline significance (0.062). We show that metastasis-associated gene 1 expression is significantly associated with malignant behavior in pancreatic endocrine tumors. This may suggest a potential role for metastasis-associated gene 1 in the malignant progression and metastasis and its use as biomarker for malignant pancreatic endocrine tumors.
\end{abstract}

Modern Pathology (2009) 22, 933-939; doi:10.1038/modpathol.2009.50; published online 17 April 2009

Keywords: MTA1; metastasis-associated gene 1; malignancy; pancreatic endocrine tumor; biomarker; metastasis

The majority of pancreatic endocrine tumors are well-differentiated neoplasms that progress slowly. According to the 2004 World Health Organization (WHO) classification, well-differentiated benign and malignant pancreatic endocrine tumors are indistinguishable based on morphological features alone.

Correspondence: Dr MD Hofer, MD, PhD, Department of Pathology, Brigham and Women's Hospital, 75 Francis Street, Boston, MA 02115, USA.

E-mail: mhofer@partners.org

${ }^{3}$ These authors contributed equally to this work.

Received 15 October 2008; revised and accepted 17 December 2008; published online 17 April 2009
Parameters such as tumor size, angio- and perineural invasion, mitotic rate, and Ki-67 proliferative index are associated with an adverse outcome; however, only unequivocal evidence of metastasis warrants a malignant classification.

The molecular basis of malignant progression in pancreatic endocrine tumors remains unknown. The metastasis-associated protein 1 (MTA1) is a member of the nucleosome remodeling and deacetylation complex (NuRD) that engages in transcriptional regulation. ${ }^{1}$ MTA1 has recently been suggested to be a downstream effector of c-myc. ${ }^{2}$ The experimental overexpression of MTA1 showed that MTA1 promotes migration and invasion of carcinoma cells 
initiating and promoting metastatic spread. ${ }^{3,4}$ The expression of MTA1 has been associated with progression to the metastatic state in various malignancies, including carcinomas of the breast, prostate, esophagus, and stomach. ${ }^{5-8}$ Our group has recently shown that MTA1 is ubiquitously expressed in both benign and malignant tumors, and its expression is particularly associated with neuroendocrine differentiation. ${ }^{9}$ This suggested a potential pivotal role of MTA in the progression of neuroendocrine malignancy. In this study we investigated the association of MTA1 expression and clinicopathological parameters of pancreatic endocrine tumors. We examined whether high MTA1 expression levels as determined by immunohistochemistry can be used to predict the clinical behavior of pancreatic endocrine tumors.

\section{Materials and methods}

\section{Clinicopathological Review of Cases}

Twenty-seven patients diagnosed with a sporadic pancreatic endocrine tumor, either on resection, necropsy, or biopsy, between 1987 and 2006 at Brigham and Women's Hospital were identified by a computer-based search of electronic pathology reports. Only sporadic cases were included in this study with no other history of an endocrine neoplasm or endocrine neoplasia syndrome. Small cell carcinomas and metastatic endocrine tumors with no definite pancreatic lesion were excluded, as were cases in which materials for additional immunohistochemistry were not available. Where sampled only as biopsies (five cases), tumors were included in analysis only if a pancreatic primary tumor was identified, and if positive neuroendocrine markers were performed at the time of diagnosis. The electronic medical records were reviewed for clinical information, including patient demographics, presence of a clinical endocrine syndrome, and patient follow-up for tumor progression up to January 2007. All patient information was handled in accordance with the institutional review board regulations.

The original slides were reexamined in all cases, along with original immunohistochemical studies where available. Pancreatic endocrine tumors were considered functional if a clear hypersecretory clinical syndrome was identified at the time of resection or biopsy. Immunohistochemistry was performed to confirm an enhanced secretory isletcell population consistent with the syndrome. Tumors were classified according to the 2004 WHO criteria into four groups: (1) well-differentiated endocrine tumor, including benign behavior; uncertain behavior; (2) well-differentiated endocrine carcinoma, and (3) poorly differentiated endocrine carcinoma.

\section{Immunostaining}

Sections of $5 \mu \mathrm{m}$ thick tissue were dewaxed and rehydrated with xylene and ethanol. After immersion in $10 \mathrm{mmol} / \mathrm{l}$ citrate buffer ( $\mathrm{pH} \mathrm{6.0)}$ ), the slides underwent microwave pretreatment for $10 \mathrm{~min}$ for optimal antigen retrieval. The primary antibody against MTA1 (anti-MTA1 monoclonal (A11), sc17773, Santa Cruz Biotechnology, Santa Cruz, CA, USA) was incubated overnight in a 1:10 dilution at $4^{\circ} \mathrm{C}$. The secondary antibody was biotin labeled and was applied for $30 \mathrm{~min}$. Streptavidin-LSA amplification method (K0679, DAKO, Carpinteria, CA, USA) was carried out for $30 \mathrm{~min}$ followed by peroxidase/ diaminobenzidine substrate/Chromagen (DAB). The slides were counterstained with hematoxylin. $\mathrm{Nu}-$ clear protein expression determined by staining intensity of the immunohistochemistry was highly reproducible and was scored as negative (score $=1$ ), weak $($ score $=2)$, moderate $($ score $=3)$, or strong (score $=4$ ), using a system that has been validated earlier. ${ }^{10,11}$ Specificity of the antibody has been shown in earlier studies where it was used for MTA1 detection in tissue and also western blot analysis. $^{5,9}$ The connective tissue present on the slide served as internal negative control and showed entirely absent staining for MTA1. Immunohistochemical staining for Ki-67 was performed using a monoclonal antibody (1:200 dilution, DAKO) along with DAB amplification (DAKO).

\section{Statistical Analysis}

Associations of MTA1 and clinicopathological parameters were analyzed using Kruskal-Wallis test of variance with a significance level of 0.05 . Survival analysis was performed by the Cox regression analysis, Kaplan-Meier method and the log-rank test, using the statistical package SPSS version 11.5 (2002, SPSS Inc., Chicago, IL, USA). One case of a benign PET was an incidental diagnosis at autopsy; for statistical analysis, this patient was considered alive, with survival censored at zero time.

\section{Results}

\section{Clinicopathological Findings}

Clinicopathological and immunohistochemical correlation in 27 pancreatic endocrine tumors between the 2004 WHO classification and clinical outcome is summarized in Table 1. In total, five tumors were functional (one gastrinoma and four insulinomas). In 10 cases $(37 \%)$, tumors were considered either benign or uncertain (WHO classification 1.1 or 1.2, respectively), based on features at presentation. Among these benign/uncertain tumors, the mean age was 63 years, the male-to-female ratio was 1.5:1, and the mean size was $2.3 \mathrm{~cm}$. In 17 cases $(63 \%)$, tumors were classified as malignant (WHO 
Table 1 Patient characteristics of 27 cases of PETs

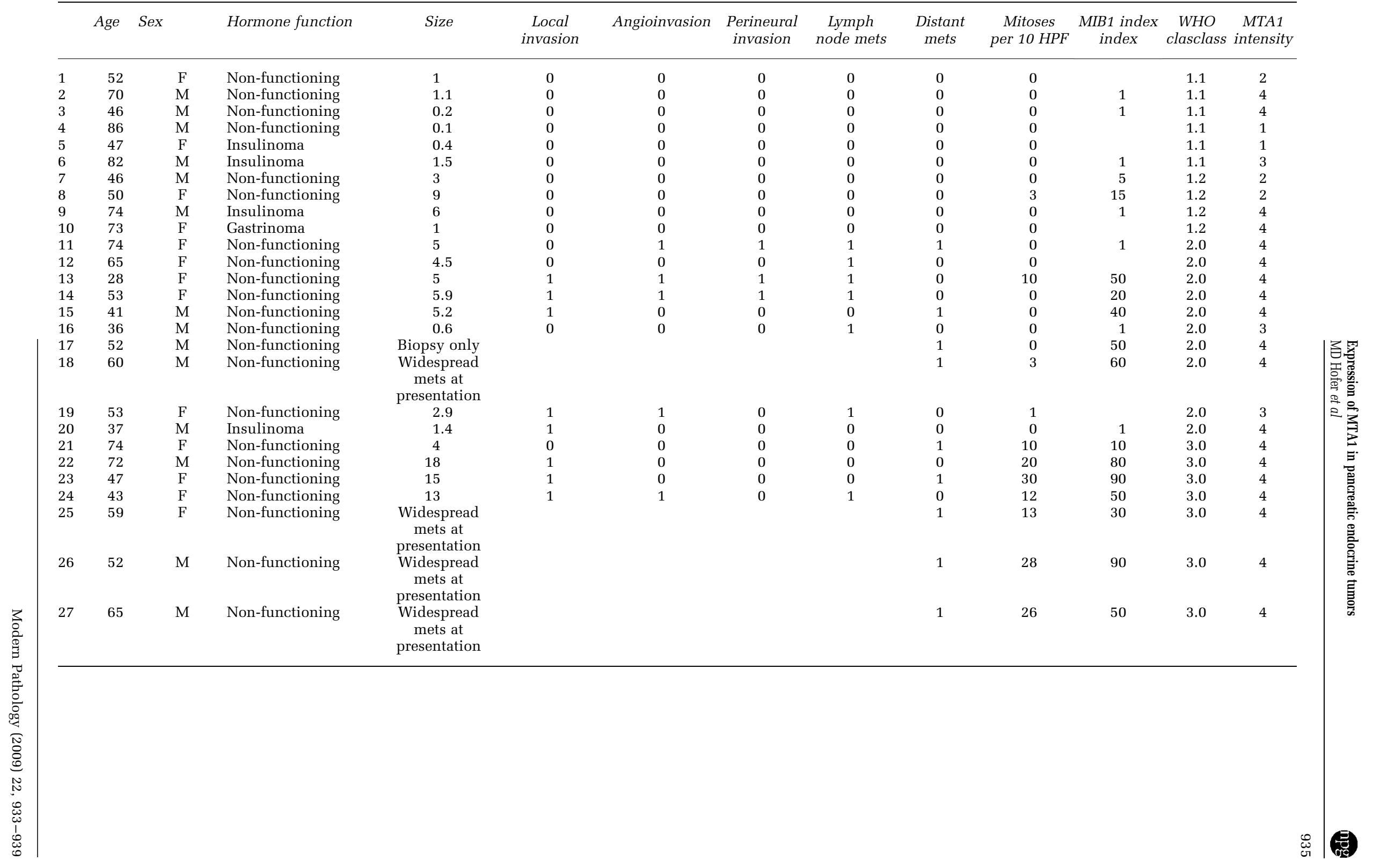


classification 2 or 3), based on local invasion and/or metastases at presentation. In this subgroup, the mean age was 54 years, the male-to-female ratio was $1: 1.1$, and the mean size was $6.7 \mathrm{~cm}$.

Well-differentiated endocrine tumors, under the WHO classification, can show either benign or uncertain behavior. Benign pancreatic endocrine tumors (WHO 1.1) comprised 6 of the $27(22 \%)$ cases. The WHO criteria that distinguish benign from uncertain (WHO 1.2) tumors are size, angioinvasion, perineural invasion, mitotic activity (2-10 per 10 high power fields) and Ki-67 proliferative activity $(>2 \%)$. Four of the $27(15 \%)$ cases were classified as having uncertain behavior, ranging in size from 1.0 to $9.0 \mathrm{~cm}($ mean $=4.8 \mathrm{~cm})$.

Malignant pancreatic endocrine tumors were classified as well-differentiated endocrine carcinoma (WHO 2) or poorly differentiated endocrine carcinoma (WHO 3). Where sampled only as metastases, tumors were included in analysis only if an earlier pancreatic primary tumor was identified, and if positive neuroendocrine markers were performed at the time of diagnosis. Well-differentiated endocrine carcinomas (10 of 27 cases, $37 \%$ ) were morphologically indistinguishable from their benign/uncertain counterparts. One of the WHO 2 tumors was an insulinoma, with otherwise benign features and no recurrence on follow-up; however, it was not confined to the pancreas, and thus was classified as having malignant behavior under the WHO criteria. By contrast, poorly differentiated endocrine carcinomas (7 of 27 cases, 26\%) were cytologically and architecturally distinct. Cytologically, tumor cells had significant mitotic activity ( $\geq 10$ mitoses per 10 high power fields), greater nuclear-to-cytoplasmic ratio, hyperchromatic nuclei, and increased variation in cell size. Necrosis was frequently present. The tumors were architecturally disordered, with predominantly solid growth. All poorly differentiated pancreatic endocrine tumors had a malignant clinical course.

\section{MTA1 Immunohistochemical Staining}

The MTA1 immunohistochemical staining in sporadic pancreatic endocrine tumors varied from noneto-focal to strong and diffuse and was evaluated semiquantitatively on a 4-tier system as represented in Figure 1. The staining was generally confined to the nucleus and homogeneous among all tumor cells.

Tumors of all WHO classifications showed a range of MTA1 staining and strong MTA1 staining was seen in cases of each WHO classification. Nevertheless, average MTA1 expression level was significantly different between WHO classes $(P=0.006)$, suggesting its association with clinical behavior and worse prognosis. In detail, benign (WHO 1.1) and uncertain (WHO 1.2) tumors had average MTA1 staining scores of $2.5 \pm 0.6$ and $3.0 \pm 0.6$, respec- tively. By contrast, unequivocally malignant tumors, well differentiated (WHO 2) or poorly differentiated (WHO 3), both had average MTA1 staining scores of $3.9 \pm 0.1$ (Figure 2). Overall, malignant tumors showed significantly higher MTA staining than benign/uncertain tumors (mean of 2.7 VS 3.9, respectively, $P<0.01$ ). Of note, we did not find an association of MTA1 expression and functionality of tumors $\left(P=0.471, \chi^{2}\right.$-test $)$. We also did not find a significant association with patient survival in Kaplan-Meier or Cox regression analysis, which we attribute to the small sample number.

To analyze the association of MTA1 expression and clinicopathological parameters and to take the small numbers of cases into account we categorized absent, weak, and moderate staining intensity as low MTA1 expression $(n=8)$ and compared it with strong MTA1 staining $(n=19)$. As shown in Table 2, strong MTA1 expression was significantly associated with tumor size $\left(\chi^{2}=4.1, P=0.044\right)$, the presence of distant metastases $\left(\chi^{2}=5.5, P=0.019\right)$, uncertain or malignant WHO classification $\left(\chi^{2}=7.7\right.$, $P=0.006$ ), and high mitotic index defined as less or more than 10 mitoses per 10 high power fields $\left(\chi^{2}=4.61, P=0.032\right)$.

\section{Discussion}

The pancreatic endocrine tumors are relatively rare, and do not cause diagnostic difficulty. These tumors can be identified by using markers of neuroendocrine cells, such as synaptophysin, neuron-specific enolase, or chromogranin, as well as hormonal markers, for example, insulin, glucagon, or somatostatin. Most pancreatic endocrine tumors are well differentiated, with a diversity of histological patterns (solid, glandular, trabecular, tubuloacinar, among others), and have a typical neuroendocrine cytomorphology. These tumors usually have a benign behavior. Whereas a subset of benign appearing pancreatic endocrine tumors behaves aggressively, there are also poorly differentiated endocrine carcinomas that are not easily recognized as endocrine tumors, thus requiring the immunophenotypic characterization with neuroendocrine markers, which also show aggressive behavior. To date, there is a lack of markers or other histomorphological findings to predict the biological behavior of well-differentiated pancreatic endocrine tumors. Metastases may develop many years after surgical excision of the primary tumor; therefore, a long clinical follow-up is required. The most reliable evidence of malignant behavior is metastasis to regional lymph nodes or liver. In this study, we evaluate the expression of the MTA1 in a set of 27 well-characterized benign and malignant pancreatic endocrine tumors with clinicopathological features consistent with previously established data $^{12}$ to determine its role as predictor of biological behavior of pancreatic endocrine tumors. 

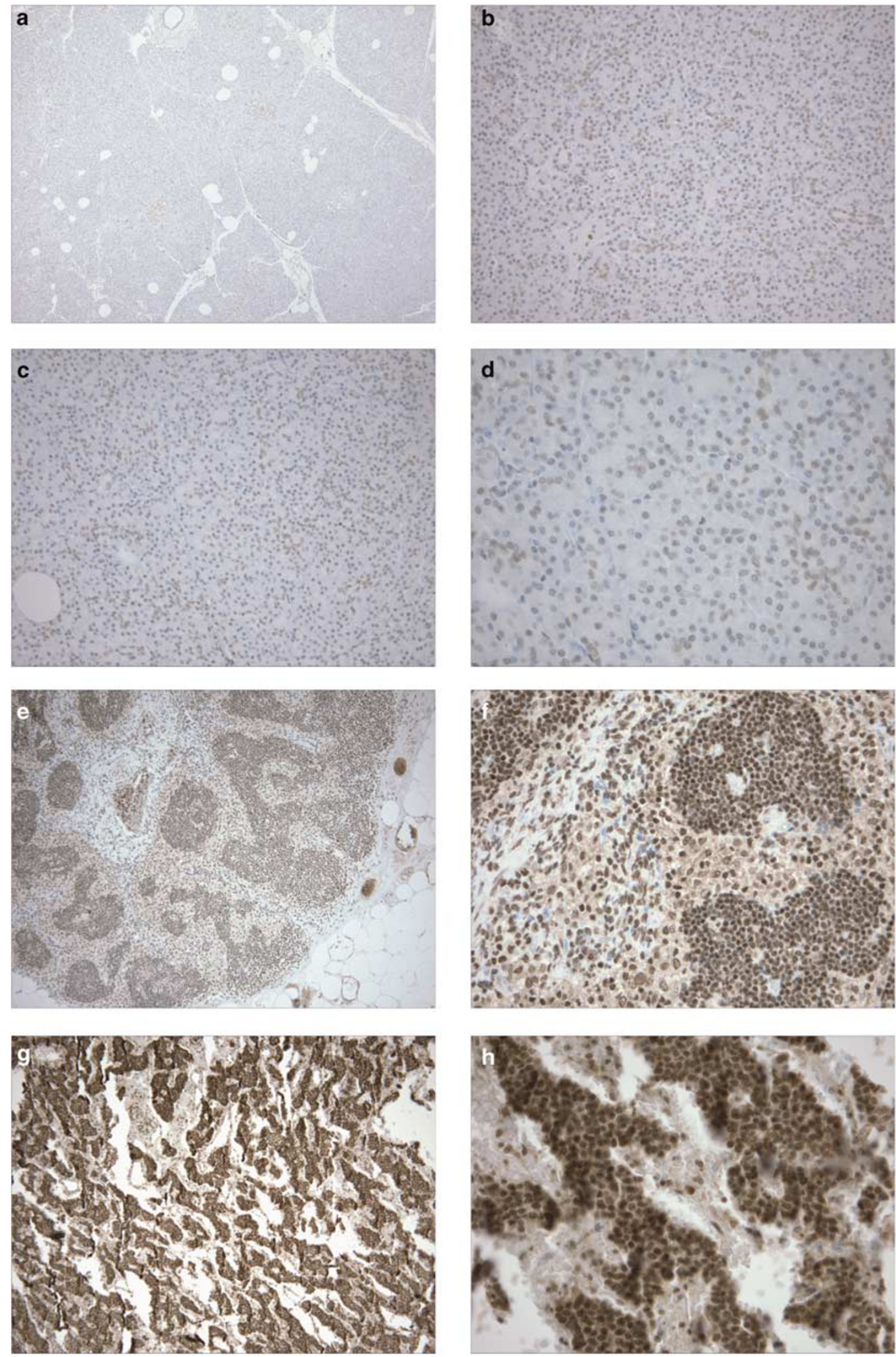

Figure 1 Absent (a and b), weak (c and $\mathbf{d}$ ), moderate (e and $\mathbf{f}$ ), and strong (g and $\mathbf{h}$ ) MTA1 staining intensity in pancreatic endocrine carcinoma in low $(\times 32)$ and high $(\times 160)$ magnification. MTA1 staining was detected using a monoclonal antibody and was confined to the nucleus. 


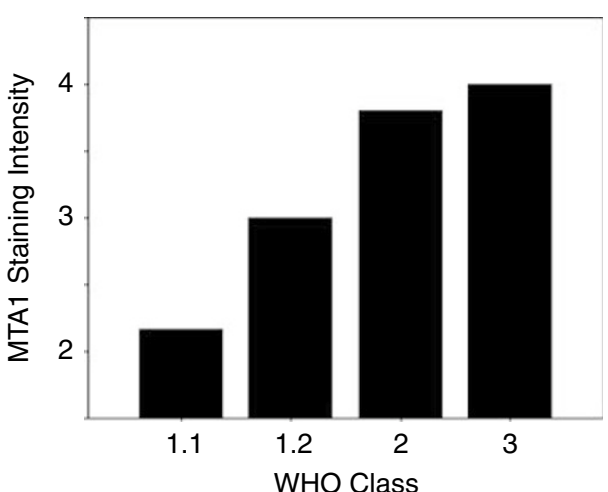

Figure 2 Graphic representation of MTA1 staining intensity evaluated semiquantitatively on a scale from 1 to 4 . Values represent the mean MTA1 staining intensity for pancreatic endocrine tumors of each WHO class.

Table 2 Association of MTA1 expression and pathological parameters

\begin{tabular}{lc}
\hline Parameter & $\begin{array}{r}\text { Significance } \\
\text { (two-tailed) }\end{array}$ \\
\hline WHO class & 0.006 \\
Age & 0.770 \\
Size (continuous) & 0.044 \\
Size ( $<$ or $>4 \mathrm{~cm})$ & 0.022 \\
Mitoses ( $<$ or 10/10 high power fields) & 0.032 \\
Ki-67 index & 0.099 \\
Local invasion & 0.086 \\
Angioinvasion & 0.398 \\
Perineural invasion & 0.169 \\
Lymph node metastasis & 0.612 \\
Distant metastasis & 0.019 \\
\hline
\end{tabular}

Our immunohistochemical studies show that MTA1 is overexpressed with significantly higher frequency in malignant pancreatic endocrine tumors. Although MTA1 was uniformly overexpressed in tumors with unequivocal malignant course, there was wide variation in MTA1 expression among benign and uncertain pancreatic endocrine tumors, with a significant proportion of benign tumors showing strong overexpression. Therefore, MTA1 is unlikely to be a discriminating marker for clinical diagnostic use. However, MTA1 expression is associated with the presence of distant metastases suggesting that this may reflect an increased propensity toward eventual metastasis. Besides representing one putative molecular step of a possible series in malignant progression, underlined by the significant association of MTA1 expression and increased mitotic rate, MTA1 could be of value as a marker of poor prognosis in the long term.

The MTA1 gene and protein were originally described in association with malignant and metastatic potential in breast carcinoma cell lines. ${ }^{13}$ MTA1 has been reported to contribute to the repression of estrogen receptor transactivation, leading to anchorage independence and hormone independence in breast cancer cells. ${ }^{14}$ It has emerged that MTA1 expression is differentially overexpressed in a variety of malignant tumors, but is also significant in selected normal tissues and benign neoplasms, particularly neuroendocrine tumors. ${ }^{9}$ Overexpression of MTA1 in both benign and malignant neoplasms of the pancreatic islet cells suggests that MTA1 alone is not sufficient to drive the tumor to metastasize. We have shown earlier that MTA1 is also overexpressed in benign paragangliomas and carcinoid tumors, suggesting that there is an association of MTA1 expression and the neuroendocrine phenotype. The exact function of MTA1 may extend from a physiological role of MTA1 in normal endocrine tissues to a pathogenic role in early neoplastic events. ${ }^{9}$ The association of MTA1 and estrogen receptor is intriguing for neuroendocrine tissues and tumors: one possible future study would be to compare MTA1 expression and hormone receptor expression in primary pancreatic endocrine tumors, or indeed in a range of endocrine neoplasms, and any subsequent metastases. One might expect loss of hormone receptor expression to be associated with increased MTA1 expression in endocrine tumors that metastasize to distant sites.

In summary, the MTA1 is a nuclear regulator of epithelial cell growth that is associated with malignant progression of epithelial malignancies, and is overexpressed in both benign and malignant neuroendocrine neoplasms. We have shown that MTA1 is overexpressed with a high frequency in malignant pancreatic endocrine tumors compared with their benign counterparts. Our results suggest that MTA1 is an important, but not a driving step in the malignant progression of pancreatic endocrine tumors. As an immunohistochemical biomarker, MTA1 may have the potential as a prognostic marker and we are currently undergoing a prospective study to further determine this.

\section{References}

1 Xue Y, Wong J, Moreno GT, et al. NURD, a novel complex with both ATP-dependent chromatin-remodeling and histone deacetylase activities. Mol Cell 1998;2:851-861.

2 Zhang XY, DeSalle LM, Patel JH, et al. Metastasisassociated protein 1 (MTA1) is an essential downstream effector of the c-MYC oncoprotein. Proc Natl Acad Sci USA 2005;102:13968-13973.

3 Hofer MD, Menke A, Genze F, et al. Expression of MTA1 promotes motility and invasiveness of PANC-1 pancreatic carcinoma cells. Br J Cancer 2004;90: 455-462.

4 Mahoney MG, Simpson A, Jost M, et al. Metastasisassociated protein (MTA)1 enhances migration, invasion, and anchorage-independent survival of immortalized human keratinocytes. Oncogene 2002;21: 2161-2170. 
5 Hofer MD, Kuefer R, Varambally S, et al. The role of metastasis-associated protein 1 in prostate cancer progression. Cancer Res 2004;64:825-829.

6 Jang KS, Paik SS, Chung H, et al. MTA1 overexpression correlates significantly with tumor grade and angiogenesis in human breast cancers. Cancer Sci 2006;97:374-379.

7 Toh Y, Kuwano H, Mori M, et al. Overexpression of metastasis-associated MTA1 mRNA in invasive oesophageal carcinomas. Br J Cancer 1999;79:1723-1726.

8 Toh Y, Oki E, Oda S, et al. Overexpression of the MTA1 gene in gastrointestinal carcinomas: correlation with invasion and metastasis. Int J Cancer 1997;74:459-463.

9 Hofer MD, Tapia C, Browne TJ, et al. Comprehensive analysis of the expression of the metastasis-associated gene 1 in human neoplastic tissue. Arch Pathol Lab Med 2006;130:989-996.

10 Lugli A, Forster Y, Haas P, et al. Calretinin expression in human normal and neoplastic tissues: a tissue microarray analysis on 5233 tissue samples. Hum Pathol 2003;34:994-1000.

11 Went PT, Lugli A, Meier S, et al. Frequent EpCam protein expression in human carcinomas. Hum Pathol 2004;35:122-128.

12 Chang MC, Xiao S, Nose V. Clinicopathologic and immunohistochemical correlation in sporadic pancreatic endocrine tumors: possible roles of utrophin and cyclin D1 in malignant progression. Hum Pathol 2007;38:732-740.

13 Toh Y, Pencil SD, Nicolson GL. A novel candidate metastasis-associated gene, mta1, differentially expressed in highly metastatic mammary adenocarcinoma cell lines. cDNA cloning, expression, and protein analyses. J Biol Chem 1994;269:22958-22963.

14 Mazumdar A, Wang RA, Mishra SK, et al. Transcriptional repression of oestrogen receptor by metastasisassociated protein 1 corepressor. Nat Cell Biol 2001;3: 30-37. 